\title{
The Influence of Wireless Network Communication and Edge Computing on the Performance of Aerobics Athletes
}

\author{
Xiaoshuang Qi (iD \\ Department of Sports, Suzhou Industrial Park Institute of Service Outsourcing, Suzhou 215000, Jiangsu, China \\ Correspondence should be addressed to Xiaoshuang Qi; qixsh@siso.edu.cn
}

Received 30 December 2021; Revised 19 January 2022; Accepted 26 January 2022; Published 24 February 2022

Academic Editor: Shalli Rani

Copyright (c) 2022 Xiaoshuang Qi. This is an open access article distributed under the Creative Commons Attribution License, which permits unrestricted use, distribution, and reproduction in any medium, provided the original work is properly cited.

\begin{abstract}
As aerobics began to be included in one of the international sports competitions, China began to pay attention to it. How to improve the performance of aerobics athletes has become a top priority. This article aims to study how to improve the performance of aerobics athletes through some high-tech techniques. To this end, this article proposes a method of combining wireless network communication and edge computing algorithms to obtain aerobics athletes' bodybuilding actions through wireless network communication and use edge computing to conduct a comprehensive analysis of related data. At the same time, experiments were designed to explore its performance and actual use effects. The experimental results of this article show that the improved performance of the athletes has increased by $13 \%$, which can help aerobics athletes to establish an advantage in terms of performance.
\end{abstract}

\section{Introduction}

Competitive bodybuilding operation is an international competitive sports project, which has a history of more than 30 years since it sprouted in the United States in the early 1980s and was introduced to my country in the late 1980s. Although competitive aerobics started late, it has developed rapidly. With the reform of the competition mechanism, the continuous reduction of the competition gap between athletes, the continuous improvement of the level of competition and the intensification of competition. For athletes of equal strength, the success or failure of the game is often determined by the athlete's competitive state during the game. Judging from the achievements of the Chinese aerobics team in the World Cup series held in this year in 2012, due to the lack of experience in major competitions among the young players born in the 90s in China, the cooperation between collective projects is insufficient, the completion of the new set is not skilled enough, and the completion of difficult skills is not stable enough. So the results of the competition are not satisfactory. This series of reasons can be attributed to the instability of the athletes' prematch competitive state, especially the psychological competitive state, which affects their own performance in the competition.

Most experts, scholars, coaches, and athletes have recognized and valued the influence and role of athletes' competitive state in the competition. At present, most of the research on "competitive state" discusses its concept, content classification, characteristics, regulation, etc. from a qualitative perspective. The research on the competitive status of athletes in different sports is also mainly focused on the research of training, physical function or psychology, and the research on the competitive status of competitive aerobics is also limited to the psychological aspect. Moreover, these studies are basically the researcher's judgment on the broad sense of discussion and experience, lack of objective and unified standards, and there is no quantitative analysis of the precompetitive state of a specific sport, which will be more meaningful to guide practice.

After the use of the computing power of edge computing to help more aerobics athletes to enhance their liquidity was proposed, more and more people began to invest in this research. Ma proposed that when organizing aerobics, each 
movement should be carefully designed to make the choreographed movements have a comfortable and pleasant feeling. The movements do not look too rigid, giving people an overall sense of beauty and aerobic exercise. The characteristics are expressed in the design of the action to achieve better results. His research found that the current aerobics combines the knowledge of dance and music, and its manifestations are diverse [1]. Cunha et al. proposed that water aerobic exercise is widely recommended to the elderly. Therefore, he evaluated the effect of water aerobic exercise on the blood pressure of elderly women with hypertension. A heart rate monitor is used to monitor the heart rate, and a semiautomatic monitor is used to measure systolic blood pressure (SBP) and diastolic blood pressure (DBP) before and after training and 10,20, and 30 minutes after training [2]. Taleb et al. introduced the survey of MEC, focusing on basic key enabling technologies. He elaborated on the MEC orchestration, while considering a single service and the MEC platform network that supports mobility, bringing brilliance to different orchestration deployment options. In addition, he analyzed the MEC reference architecture and main deployment scenarios to provide multi-tenant support for application developers, content providers and third parties [3]. Ke et al. proposed a cloud-based mobile edge computing (MEC) offloading framework in vehicle networks. In this framework, he studied the effectiveness of the calculation transmission strategy for vehicle-to-infrastructure (V2I) and vehicle-to-vehicle (V2V) communication modes. Taking into account the time consumption of computing tasks and the mobility of vehicles, he proposed an effective predictive combination mode degradation scheme in which tasks are adaptively offloaded to the MEC server through direct upload or predictive relay transmission [4]. He et al. first introduced the deep learning of the Internet of Things to the edge computing environment. Due to the limited processing power of existing edge nodes, he also designed a novel offloading strategy to optimize the performance of IoT deep learning applications through edge computing. The evaluation results show that his method is superior to other optimization solutions in terms of deep learning of the Internet of Things [5]. Yu et al. proposed that the Internet of Things (IoT) now penetrates into our daily lives, providing important measurement and collection tools for every decision we make. Compared with the well-known cloud computing, edge computing migrates data computing or storage to the "edge" of the network, close to the end users. Therefore, multiple computing nodes distributed on the network can offload the computing pressure from the centralized data center and can significantly reduce the delay of message exchange [6]. Nastic et al. propose a novel method to implement cloud-supported real-time data analysis in edge computing applications. He introduced their serverless edge data analysis platform and application model, and discussed their main design requirements and challenges based on real healthcare use case scenarios [7]. Chen et al. designed a resource-efficient edge computing solution so that users of smart IoT devices can well support their computing-intensive tasks through proper task offloading of local devices, nearby auxiliary devices, and nearby edge clouds. Performance evaluation confirmed the effectiveness and superior performance of the proposed resourceefficient edge computing scheme [8]. The abovementioned documents mainly introduced related edge computing algorithms, expressiveness of aerobics, and wireless communication networks. It is also very in place for their respective technical descriptions, and the depth of research on some technologies is also worth learning. However, there is no literature that discusses the combination of these types of research and conducts related research on them.

The innovation of this article is to use wireless network communication and edge computing as the technical support to conduct a comprehensive collection of aerobics athletes' bodybuilding movements. And use the computing power of edge computing to analyze and guide it, helping aerobics athletes to improve related actions and enhance their performance. At the same time, experiments and analysis are designed to test the calculation performance and data acquisition ability and to explore the range of improving aerobics athletes.

\section{Wireless Sensor Communication Method}

\subsection{Wireless Sensor Network}

2.1.1. The Overall Structure of the Wireless Communication System. The overall structure of the system is mainly composed of three parts (ZigBee terminal node, gateway, and host computer) as shown in Figure 1 [9]. The terminal node can be connected with the workshop or factory area meter, the home appliance equipment in the smart home, and so on, and read and write the data of the meter or equipment through the serial port and upload it to the gateway through the ZigBee wireless network [10]. The gateway is the core controller of the entire ZigBee network, so as to realize the communication between the upper computer and the underlying equipment (mainly responsible for the network establishment and maintenance of the entire network and responsible for the protocol conversion between Ethernet and ZigBee networks). The gateway includes a ZigBee coordinator, a WiFi module, a wireless router, an Ethernet interface, and other modules [11]. Among them, the WiFi module and the Ethernet interface, respectively, provide wireless and wired modes to communicate with the host computer; the built-in wireless router in the gateway is responsible for establishing the $\mathrm{WiFi}$ network; the ZigBee coordinator is responsible for setting up a ZigBee network to realize data exchange with ZigBee terminal nodes. A BOA server is built in the gateway, and users can access the gateway through the Web and configure the network. The upper computer is used to configure and monitor the system and only supports three forms (desktop, notebook, and Android handheld terminal) [12].

2.1.2. Wireless Sensor Network Protocol. As shown in Figure 2, the communication protocol layer can be divided into the physical layer, the link layer, the network layer, the transport layer, and the application layer. The network management plane can be divided into the energy 


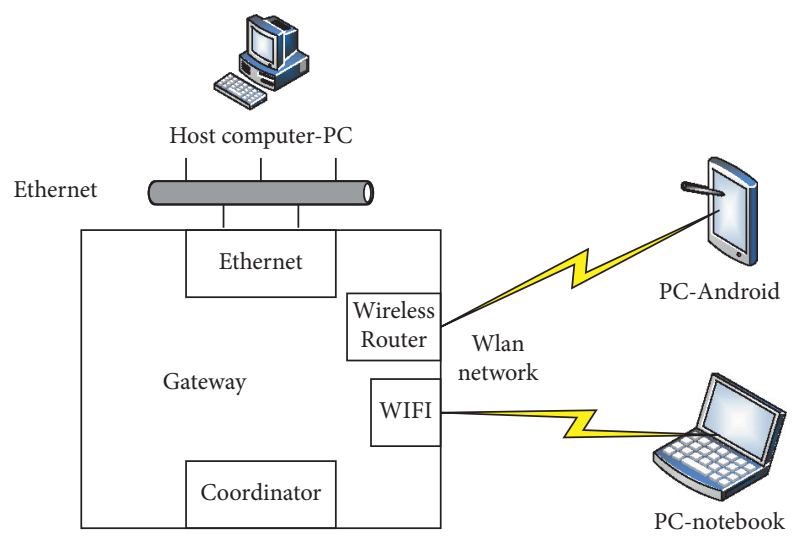

FIgURE 1: The overall structure of the system.

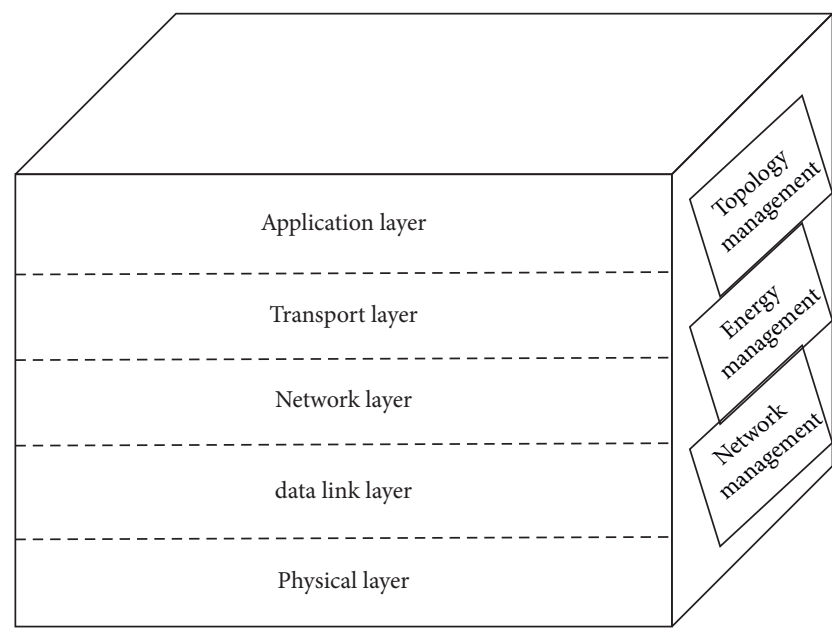

FIGURE 2: Wireless sensor network protocol stack structure diagram.

management plane, the mobility management plane, and the task management plane [13].

The wireless sensor network is divided into three structures (star, mesh, and hybrid networks). The star network is a single-hop network, and all terminal nodes can exchange data with routers and gateways. The mesh network is a multihop network, and all terminal sensor nodes can directly transmit data in both directions and can also communicate with the base station [14]. The hybrid network not only has the advantages of low power consumption and simplification of the star network but also has the advantages of long-distance transmission and automatic repair of the mesh network [15].

2.1.3. Wireless Node Hardware Design. The wireless node hardware structure includes a CPU part, a reset circuit part, a clock circuit part, a radio frequency antenna part, a power circuit part, and a data acquisition part. As shown in Figure 3, the data acquisition part provides a serial port to communicate with the test instrument [16]. The wireless node adopts the on-board antenna and reserves an external antenna interface. Also consider anti-interference issues in

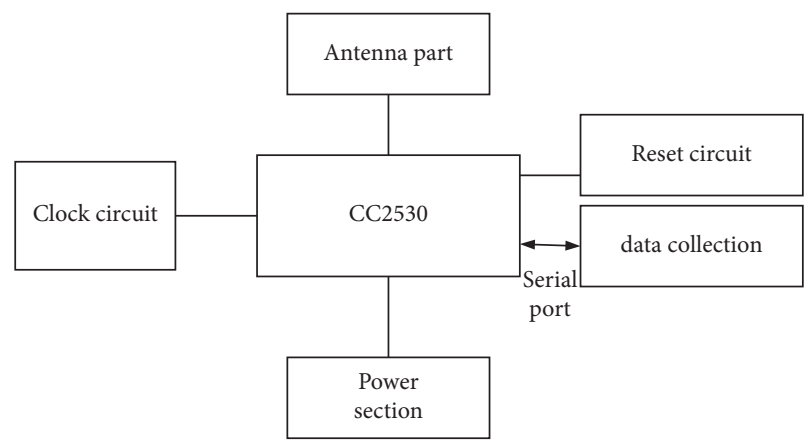

Figure 3: Wireless node hardware structure.

hardware design, and try to avoid interference between components and lines through reasonable layout and wiring [17].

2.1.4. Wireless Gateway Hardware Design. According to the application requirements of the wireless gateway, the hardware structure of the gateway is shown in Figure 4. Among them, the ZigBee wireless node adopts the same hardware design as the wireless gateway [18]. In view of the hardware and software functional requirements of this data server, the core controller adopts Samsung processors, and the hardware modularization is divided into two-layer board structures (core board and bottom board), which is convenient for design and maintenance [19].

The core controller uses Samsung processors, and the gateway uses ARM processors (compared with other 8-bit or 16-bit single-chip microcomputers, it has a faster processing speed and at the same time makes the entire system have better scalability) [20]. The ARM processor occupies a pivotal position in the embedded field with its high-speed data storage speed, flexible development tools, powerful instruction system, and a wide range of applications [21].

2.2. Communication Model. In the mobile edge computing system, communication mainly occurs between the mobile device and the MEC server. The MEC monthly server is a small data center deployed by cloud computing service providers or operators on wireless access points such as base stations and routers. Wireless access points not only provide wireless communication air interfaces for mobile devices and MEC servers, but also connect to large data centers located in the core network. It can help MEC servers to further offload computing tasks to large data centers or other MEC servers [22].

Some commercial wireless communication technologies mainly include NFC, Bluetooth, WiFi, and LTE. These wireless communication technologies can support the communication requirements of mobile devices and MEC servers at different transmission rates and different distances. The table lists the main characteristics of these typical wireless communication technologies [23]. For example, maximum coverage, frequency spectrum, and data transmission rate. NFC technology has a small coverage area and 

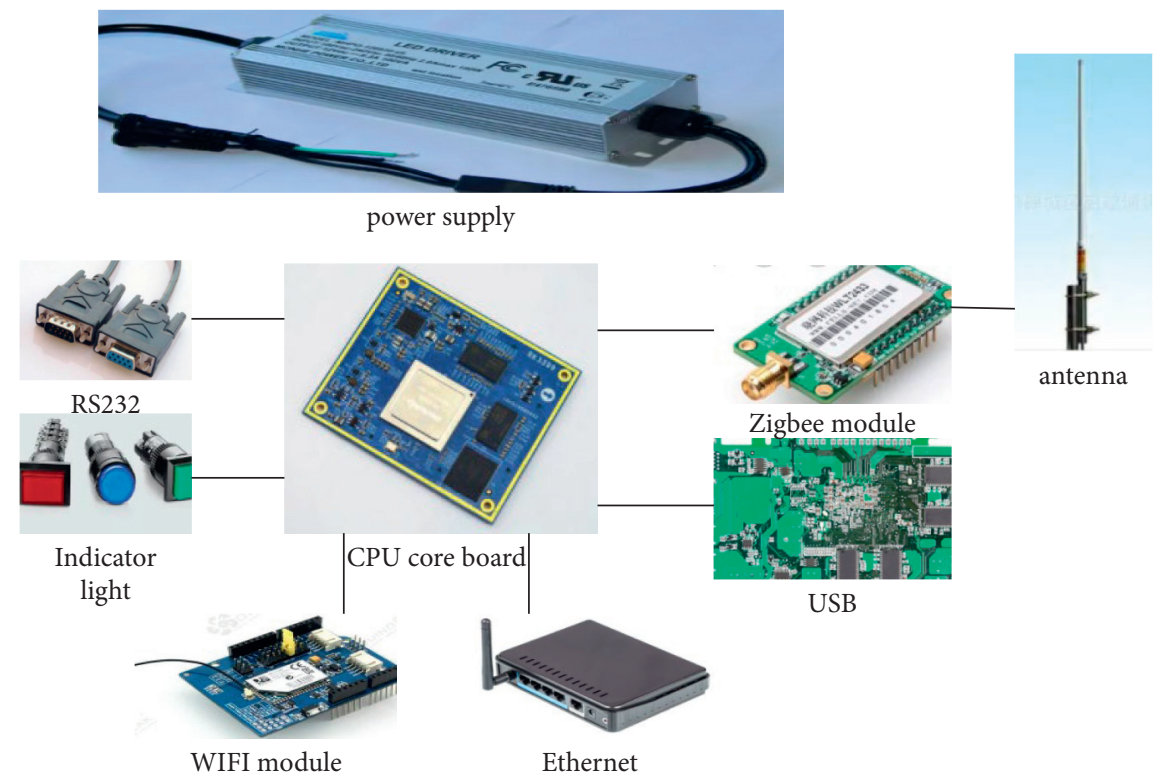

FIGURE 4: Wireless gateway hardware structure.

a low data transmission rate, which is suitable for services that require a small amount of information exchange, such as payment services [24]. RFID is similar to NFC, but only supports simplex communication. Compared with NFC and RFID, Bluetooth technology has a higher transmission rate, which can make the communication between mobile devices and MEC servers faster and more efficient in short-distance scenarios [25]. In long-distance scenarios, WiFi and LTE are two very effective communication methods. Related technical parameters are shown in Table 1.

Based on the several wireless communication methods listed in this section, according to Shannon's formula, the maximum data transmission rate at which the mobile device sends the data volume of the subtask to the MEC server is obtained, as shown in the following formula:

$$
R=B * \log _{2}\left(1+\frac{H P_{t r}^{i}}{\delta^{2}}\right) .
$$

For mobile devices, computing performance is determined by CPU performance, and CPU performance is controlled by CPU frequency. The locally calculated time is shown in the following formula:

$$
t_{c p}^{i O}=\frac{D_{i} * X_{i}}{f_{o}} .
$$

Mobile devices are battery-powered and energy-constrained devices. Therefore, when dealing with computationally intensive tasks, the battery energy consumed is a very important consideration. The energy consumed by the subtask calculation on the mobile device can be expressed as the following formula:

$$
E_{c p}^{i O}=k_{o} D_{i} X_{i} f_{0}^{2}
$$

According to Shannon's formula, the maximum transfer rate from a mobile device to the MEC server is as follows:

$$
R_{j}=B * \log _{2}\left(1+\frac{H P_{t r}^{i}}{\delta^{2}}\right)
$$

The transmission energy consumption is equal to the transmission power multiplied by the transmission time, as shown in the following formula:

$$
\begin{aligned}
& t_{t r}^{i j}=\frac{D_{i}}{R_{j}}, \\
& t_{t r}^{i j}=\frac{D_{i}}{B * \log _{2}\left(1+\left(H P_{t r}^{i} / \delta^{3}\right)\right)}, \\
& E_{t r}^{i j}=P_{t r}^{i} * t_{t r}^{i j}, \\
& E_{t r}^{i j}=\frac{D_{i}}{B} * \frac{P_{t r}^{i}}{\log _{2}\left(1+\left(H P_{t r}^{i} / \delta^{2}\right)\right)} .
\end{aligned}
$$

The energy consumed is shown in the following two formulas:

$$
\begin{gathered}
t_{c p}^{i j}=\frac{D_{i} * X_{i}}{f_{i}}, \\
E_{c p}^{i j}=k_{j} D_{i} X_{i} f_{j}^{2} .
\end{gathered}
$$

The optimization goal of this chapter is to find a solution for offloading decision-making and power allocation, which minimizes the system energy consumption of a certain separable computing task under a given time limit. The offloading decision and power allocation scheme refer to the MEC server to which several independent subtasks on the mobile device should be offloaded for execution or should be left on the local device for execution, and how much transmit power the mobile device should allocate for each subtask. The problem can be formulated as formulas: 
TABLE 1: Comparison of wireless communication technologies.

\begin{tabular}{lccccc}
\hline & NFC & RFID & Bluetooth & WiFi & LTE \\
\hline Coverage distance & $10 \mathrm{~cm}$ & $3 \mathrm{~m}$ & $100 \mathrm{~m}$ & $100 \mathrm{~m}$ & $100 \mathrm{~km}$ \\
Frequency & $13.56 \mathrm{MHz}$ & $13.56-960 \mathrm{MHz}$ & $2.4 \mathrm{GHz}$ & $5 \mathrm{GHz}$ & $700-2600 \mathrm{MHz}$ \\
Rate & 414 & $100-200 \mathrm{~B} / \mathrm{s}$ & $22 \mathrm{Mbps}$ & $135 \mathrm{Mbps}$ & $300 \mathrm{Mbps}$ \\
\hline
\end{tabular}

$$
\begin{aligned}
& \min _{a, p} \sum_{i=1}^{N}\left(E_{t r}^{i}+E_{c p}^{i}\right), \\
& t_{t r}^{i}+t_{c p}^{i} \leq \tau, \quad \forall i \in\{1,2,3 \ldots N\}, \\
& \sum_{i=1}^{N} P_{t r}^{i} \leq P_{\max } .
\end{aligned}
$$

Assigning subtasks to a device for calculation has a certain cost. The physical meaning of edge weight is the cost of energy consumption (transmission energy consumption + computing energy consumption). In particular, for a certain subtask, if the offloading decision is calculated locally on the mobile device, then the edge weight only includes the calculation energy consumption. It was shown as the formula for details.

$$
\begin{aligned}
& \text { edge }[i][j]=E_{c p}^{i 0}, j=0, \\
& \operatorname{edge}[i][j]=E_{t r}^{i j}+E_{c p}^{i j}, \quad j !=0 .
\end{aligned}
$$

This algorithm is based on depth-first search or breadthfirst search and has low computational complexity. In the simulation data, it is found that the transmission energy consumption is far less than the calculated energy consumption, and its order of magnitude is very small. Therefore, it can be approximated as follows:

$$
\text { edge }[i][j]=E_{c p}^{i j} \text {. }
$$

In the previous step, the calculation of total energy consumption has been minimized, and the problem becomes as follows:

$$
\min \sum_{i=1}^{N} E_{t r}^{i}=\sum_{i=1}^{N} \frac{D_{i}}{B} * \frac{p_{t r}^{i}}{\log _{2}\left(1+H P_{t r}^{i} / \delta^{2}\right)} .
$$

The constraints remain unchanged.

First, create the function as follows:

$$
f(x)=\frac{x}{\log _{2}(1+A x)} \text {. }
$$

The first derivative of $f(x)$ is as follows:

$$
f^{\prime}(x)=\frac{\log _{2}(1+A x)-(A x /((1+A x) \ln 2))}{\log _{2}^{2}(1+A x)} .
$$

It can be proved that when $x>0, f^{\prime}(x)>0$. Therefore, $f(x)$ increases as $\mathrm{x}$ increases. Therefore, we can get the analytical solution as follows:

$$
P_{t r}^{i}=\frac{\left(2^{D_{i} /\left(\tau-t_{c p}^{i}\right) B}-1\right) \sigma^{2}}{H_{i}} .
$$

Among them, $B$ is the line channel bandwidth, $H$ is the channel gain, and $P_{t r}^{i}$ is the transmit power allocated by the mobile device for the subtask. $D_{i}$ and $X_{i}$ represent the total number of time cycles required to process subtasks. $f_{0}$ is the $\mathrm{CPU}$ frequency of the mobile device. $\delta^{2}$ is the noise power.

2.3. Mobile Edge Computing Network Design. At present, most of the work related to mobile edge computing in the world is in the research stage. According to the future development trend of the access network and the basic needs of users for the access network, this paper mainly studies the mobile edge computing networking model. It designs a mobile edge computing platform architecture that integrates access networks and cloud computing capabilities and conducts related functional tests. This chapter will elaborate on these contents.

The application scenario of the mobile edge computing architecture is shown in Figure 5. Mobile users establish connections with the mobile edge network through base stations (such as LTE macro base stations, 3G wireless network controllers, and multitechnology convergence access points). The edge server is deployed at the location closest to the base station equipment and is physically connected to the base station equipment. The edge server has the ability to process and control user data traffic at the same time. The user's request and network message are sent to the local MEC server through the wireless access network. On the edge server, there are forwarding and filtering rules deployed by mobile network operators to control user traffic, as well as applications and services from third-party service providers. Therefore, at the edge of the mobile network, the edge server can both process and forward the user's request. After the edge server receives the user request from the wireless access network, it makes a corresponding response. When the local server can handle the user request, it can directly provide the corresponding cloud service. When the corresponding service is not used in the local edge server, the request can be forwarded to the edge server in the neighboring area, or the user request can be directly forwarded to the core network for processing.

In traditional mobile cloud computing, user requests and information are first transmitted to the central processing unit that provides mobile network access services. After being authenticated and authorized by the network operator, it is forwarded to the cloud data center via the mobile core network to obtain different services (such as databases, virtualized 


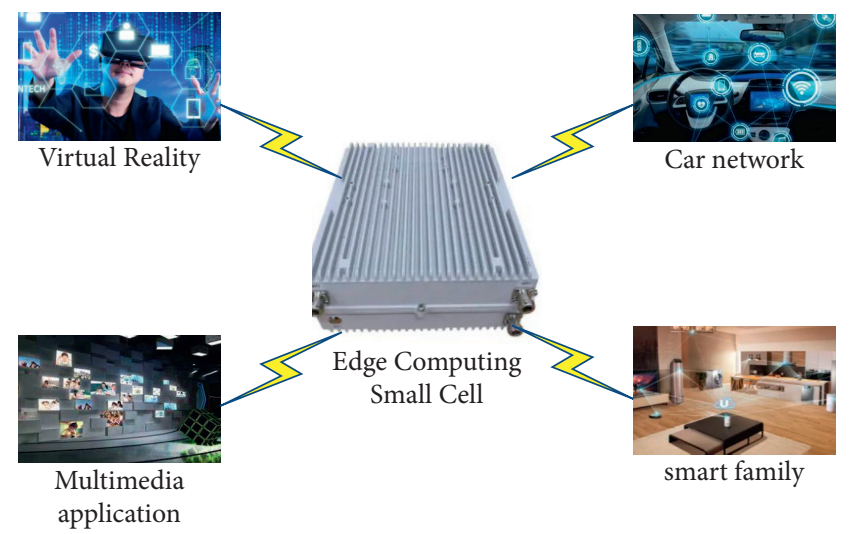

Figure 5: Schematic diagram of mobile edge computing application scenarios.

resources, computing resources, and storage services). Different from mobile cloud computing, in the mobile edge computing architecture, the functions of the cloud data center are migrated to the edge of the mobile network, and they directly process and respond to user requests on the wireless access network. Using distributed edge servers and wireless access networks, users can support a variety of applications, for example, high-definition video distribution, augmented reality, human-computer interaction, location services, data caching, mobile big data analysis, etc.

2.3.1. MEC Platform Architecture. With the continuous expansion of the influence of SDN and NFV, as well as the further development of cloud computing and edge computing, the softwareization of telecommunications and network systems has become a key promotion factor. In order to meet the user needs of next-generation networks and the basic requirements of ICT convergence, the software architecture of mobile computing and edge network convergence has been extensively studied in industry and academia.

The basic relational model of mobile edge computing proposed by ETSI is shown in Figure 6. In order to meet the needs of system management and scalability, the cloud platform adopts a layered structure to realize the virtualization of physical resources and the unified management of virtualized resources. At the same time, the mobile edge computing platform should also provide an interface to manage the entire platform. The operator manages the application platform, application life cycle, and other operations on the application through this interface. In addition, according to the requirements of mobile edge computing, the edge cloud platform also provides infrastructure services (communication services and service registration), wireless network information services, and traffic offloading services.

(1) Communication Services. Through a specially designed system interface, applications running on the platform can communicate with each other through infrastructure services. In addition, infrastructure services also support communication between applications and platforms. The communication service module can achieve the purpose of one-to-many message publishing and application decoupling, and at the same time can provide a protection mechanism to defend against malicious applications.

(2) Service Registration. Provide a list of service types supported by the edge server, and provide flexible deployment strategies for applications through decoupling. In addition to the service list, the service registration module also provides related interfaces and their versions. Applications can use it to discover and locate required service locations and broadcast their own location to provide services to other applications.

(3) Wireless Network Information Service. Mobile edge computing allows applications running on edge servers to obtain real-time network and wireless information. The wireless network information service module in the platform can provide corresponding edge network information for certified applications. Third-party applications can perform high-level processing based on this open network information. The information provided by this module is mainly user equipment access-related information (such as QoS and cell ID), user-related measurement, and statistical information.

(4) Traffic Offloading Module. The traffic offloading module can control the traffic on the entire wireless access network at the data packet level. The traffic of those authenticated applications can be prioritized by setting rules. It plays an important role in the flow balance and QoS of the entire system.

\subsubsection{The Key Realization Technology of MEC Networking} Scheme. Network function virtualization, software-defined networking, and edge operating systems are key technologies in the implementation of mobile edge computing networking. Network function virtualization technology can realize the orchestration and management of computing and storage resources in mobile edge computing; software-defined network technology can realize the programming of edge network resources; and the edge operating system ensures the scalability of the entire mobile edge computing platform and speeds up the deployment of edge cloud.

Through the layered structure, the edge operating system can realize the abstraction and flexible allocation of physical resources such as computing, storage, and networking. At the same time, the recursive structure ensures the scalability and expansibility of the edge operating system and can flexibly control the scale and coordination of edge data centers.

\section{Edge Computing Platform Performance Test Experiment}

3.1. Performance Test. In this section, we will discuss and analyze the performance of the edge computing platform. It mainly includes network forwarding delay performance, 


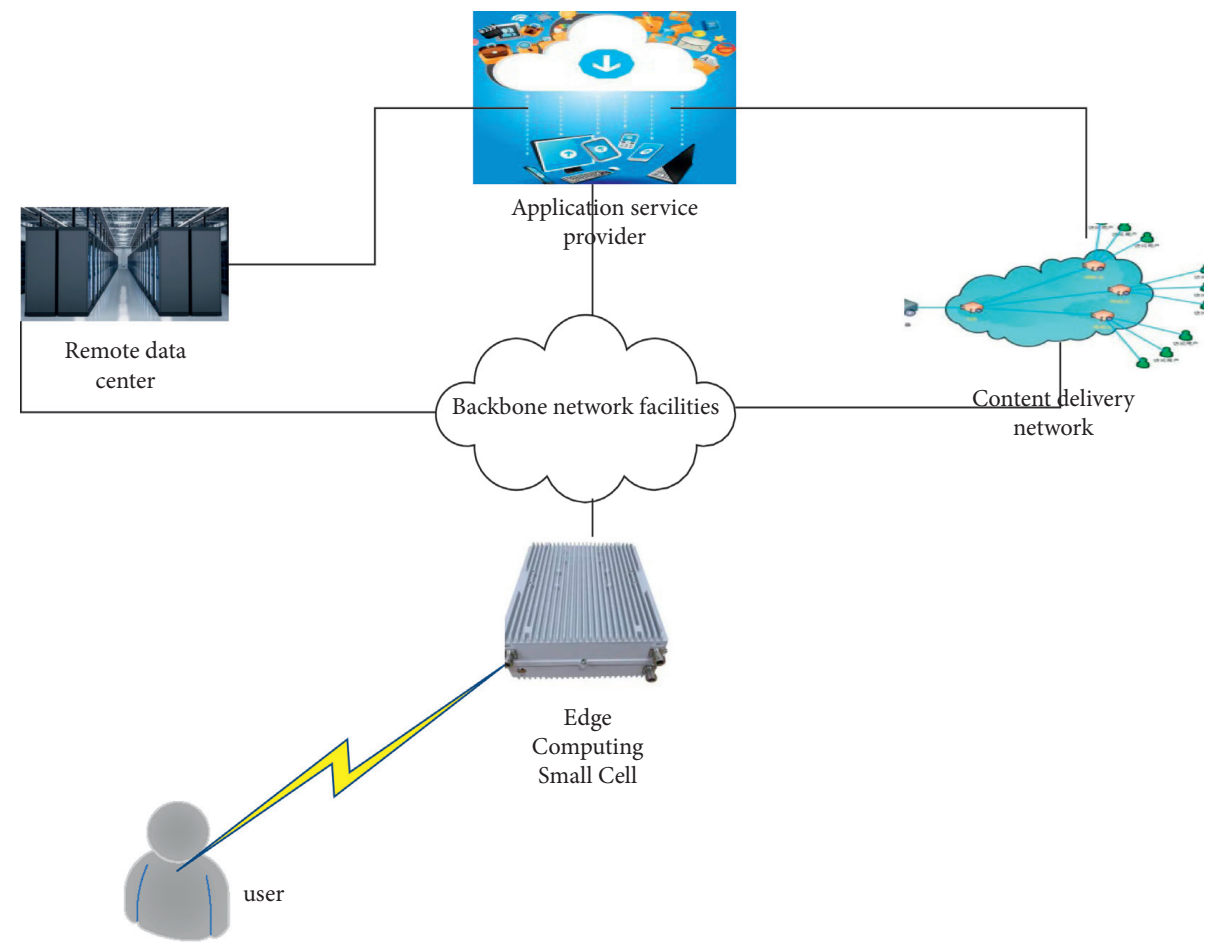

FIgURE 6: The basic relational model of mobile edge computing.

request processing capacity, power consumption performance, migration efficiency, and performance evaluation of container service orchestration. In each experiment, we introduced in detail the experiment preparation, the experiment process, and the experiment result analysis. In our experimental environment, the main equipment configurations used are shown in Table 2.

First of all, regarding the network forwarding delay, it will compare the network packet transmission round-trip time (RTT) of the EIS with that of the OvS installed with commercial SDN switches (CentecV350-8TS12X) and wireless routers. Two EIS devices with different hardware architectures were used during the experiment: the Raspberry3B single-board computer based on the ARM architecture and MinnowBoard Turbot single-board computer based on the $x 86$ architecture. The prices of the two devices are about RMB280 and RMB2000, respectively. This comparison can not only analyze the performance pros and cons of platform EIS nodes compared to commercial switching equipment but also select node types within the platform cluster based on cost factors. It is worth pointing out that because DPDK running on Raspberry3B will bring equipment performance bottlenecks, we did not install DPDK on Raspberry, but instead only implemented OvS + DPDK practice on Turbo devices. In the experiment, we connect the two hosts through the abovementioned four different switching devices to form a single-hop network connection. Several experiments were performed during the measurement process, and the ping command of the Linux system was used to send five different data packet sizes from one host to another host for testing, recording the delay result, and calculating the average value. The specific values are shown in Table 3.
The results show that Turbot can reach the level of commercial SDN switches due to its own Gigabit Ethernet interface, combined with the optimization of DPD1C technology. When the data packet size is increased from 64 bytes to 1024 bytes, it has better stability. When pursuing EIS nodes with high network performance in the edge network environment, Turbot will be the first choice. Although the network forwarding performance of Raspberry equipment is not as good as that of commercial switches, it is significantly better than ordinary routing and switching equipment. It is especially suitable as a lowcost network node pursuing high cost performance in the Internet of Things scenario. It cannot be ignored that the network switching nodes implemented by the platform all support the OpenFlow protocol, which can extract the control logic to the SDN controller for unified management. While EIS completes the network switching function, it can still carry the function of the service node at the same time, which is impossible for ordinary network equipment.

Request processing capacity due to the hardware difference between EIS and rack servers. The processing power of a single EIS is far inferior to that of rack servers. We designed experiments to compare the request processing capabilities of EIS single node, EIS cluster, and rack server (DELLR720) through service request response time. During the experiment, we used 8 EIS (Raspberry3B) devices to form a service cluster, and at the same time, we built a singlenode service on one EIS and one rack server. We installed the ApacheBench stress test tool on the client to simulate 100 users to send 1000 data requests to single EIS, 8EIS clusters, and rack servers (request file size is $4 \mathrm{MB}$ ), and record the service response time. 
TABLE 2: Test environment specific configuration.

\begin{tabular}{lccc}
\hline Equipment & Model & Configuration & \\
\hline Mobile terminal & Xiaomi Mix & 4 GB RAM & Price \\
Edge intelligence server & Raspberry 3B & Broadcom BCM2837 & $\$ 3500$ \\
SDN switch & Centec V350-8TS12X & $8 * 1000$ Base-X & $\$ 250$ \\
\hline
\end{tabular}

TABLE 3: Comparison of network forwarding delay.

\begin{tabular}{lcccc}
\hline Bytes & Wireless router & Raspberry-EIS & Turbot-EIS & SDN switch \\
\hline 64 & 0.64 & 0.6 & 0.39 & 0.4 \\
128 & 0.65 & 0.61 & 0.41 & 0.38 \\
256 & 0.67 & 0.63 & 0.4 & 0.4 \\
512 & 0.68 & 0.65 & 0.47 & 0.41 \\
1024 & 0.85 & 0.65 & 0.58 \\
\hline
\end{tabular}

The experimental results show that the response time of a single EIS is quite different from that of a rack server, and $10 \%$ of requests will not get a better quality of experience. However, using 8 EIS to process experimental requests in a distributed manner can achieve a response time similar to that of the rack server, and the overall difference is small. It is worth emphasizing that the price of a commercial server is about RMB 16000, and the cost of 8 EIS is about $1 / 7$ of that of a commercial server. We achieved service request processing performance similar to that of commercial servers at a cost of $1 / 7$. This shows that when processing computing tasks and requests similar to those found in the Internet of Things environment, the EIS platform can provide lightweight and cost-effective request processing capabilities at the user edge. The specific data are shown in Table 4.

3.2. Power Performance. Regarding power consumption performance, we compared EIS nodes with commercial servers (DELLR720), commercial SDN switches (CentecV350-8TS12X), and wireless routers (TP-LINKTLWDR7500). During the experiment, we used a power tester (UNI-TUT230C) for testing, connected the abovementioned equipment with the tester, and modified the CPU load through the stress test tool, and recorded the test results after multiple measurements. The specific values are shown in Table 5.

It can be seen from the abovementioned table that the CPU is idle, the CPU usage rate is $50 \%$, and the CPU usage rate is $100 \%$. The power consumption of EIS nodes is lower than commercial servers and switches and even close to wireless routers. The experimental results fully verify the low power consumption characteristics of the EIS equipment in the platform when providing services.

\section{Wireless Network Simulation Analysis}

4.1. Comparison of Average Data Packet Exchange Times of Intermediate Nodes. In the case of a given network size $(N=1200)$, Figure 7 shows the average number of data packet exchanges of the intermediate nodes of the three mechanisms under different maximum replication times value Ymax, that is, the average energy consumption of the intermediate nodes. Since the COPE mechanism and the ER mechanism are not
TABle 4: Comparison of request processing capabilities.

\begin{tabular}{lccc}
\hline & Rack server & 1 -EIS & 8 -EIS \\
\hline $50 \%$ & 0.38 & 0.42 & 3.21 \\
$75 \%$ & 0.41 & 0.43 & 3.82 \\
$90 \%$ & 0.62 & 0.67 & 4.48 \\
$98 \%$ & 0.81 & 0.98 & 5.84 \\
$100 \%$ & 0.91 & 1.12 & 7.01 \\
\hline
\end{tabular}

TABle 5: Power consumption comparison.

\begin{tabular}{lccc}
\hline & CPU idle & $50 \%$ CPU usage & 100\% CPU usage \\
\hline EIS & 4 & 6 & 9 \\
Rack server & 121 & 214 & 391 \\
SDN switch & 37 & 41 & 59 \\
Wireless router & 4 & 6 & 8 \\
\hline
\end{tabular}

limited to the number of data packet replications, the average number of data packet exchanges at intermediate nodes will not change. When the maximum copy time value is low, the average data packet exchange times of the intermediate nodes of the CRNC mechanism are much lower than those of the ER mechanism and the COPE mechanism. Therefore, the energy consumption of the intermediate node is low as the limit of the maximum number of replications is approaching the minimum number of replications required to infect the entire network Ynet. The average number of data packet exchanges of the intermediate nodes of the mechanism also gradually rises and approaches the COPE mechanism. The CRNC mechanism based on network coding can complete the duplication of multiple source-code data packets in one data packet exchange process, which improves the transmission efficiency, and the energy consumption level can always be kept under the ER mechanism.

For the comparison of transmission delays, first analyze the performance comparison of the three mechanisms in the case of unlimited energy. As shown in Figure 8, when the maximum number of replications is close to the minimum number of replications required to infect the entire network, the transmission delay of the CRNC mechanism will gradually decrease, and its performance will approach the COPE mechanism. Compared with Figure 7, when the maximum copy time value is low, for example, from 1 to 6 , compared to the COPE mechanism, the CRNC mechanism 

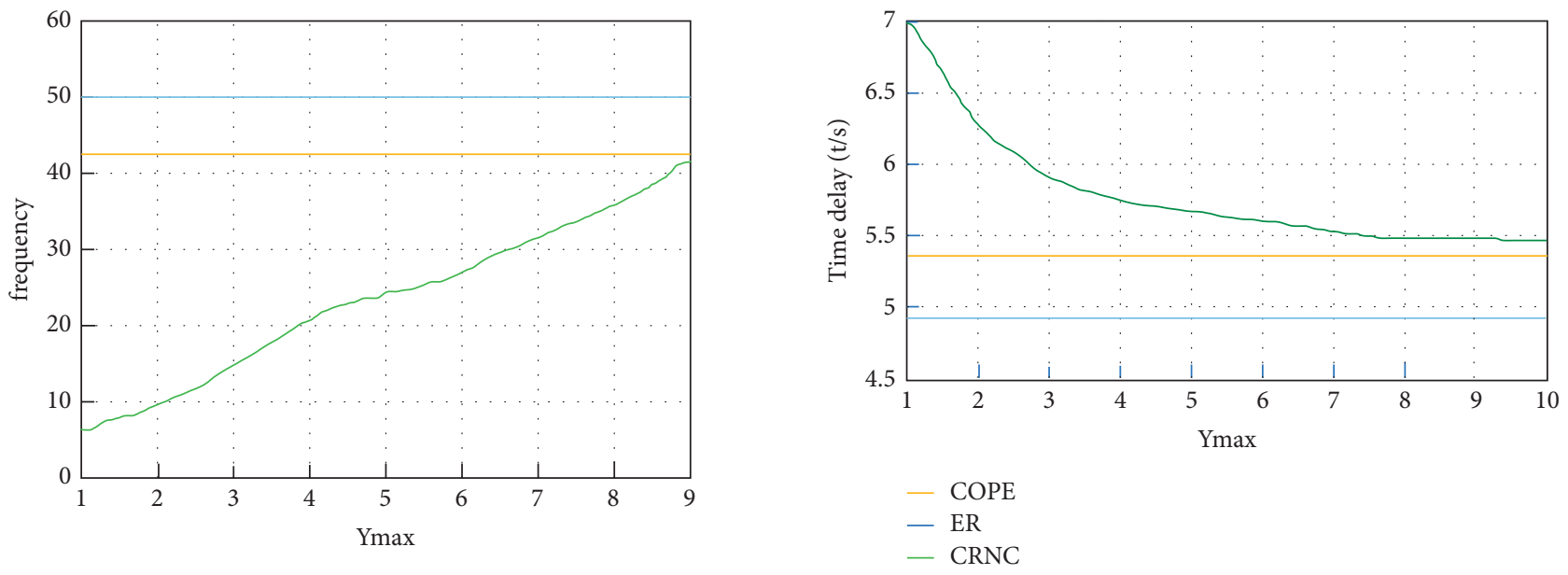

COPE

- ER

- CRNC
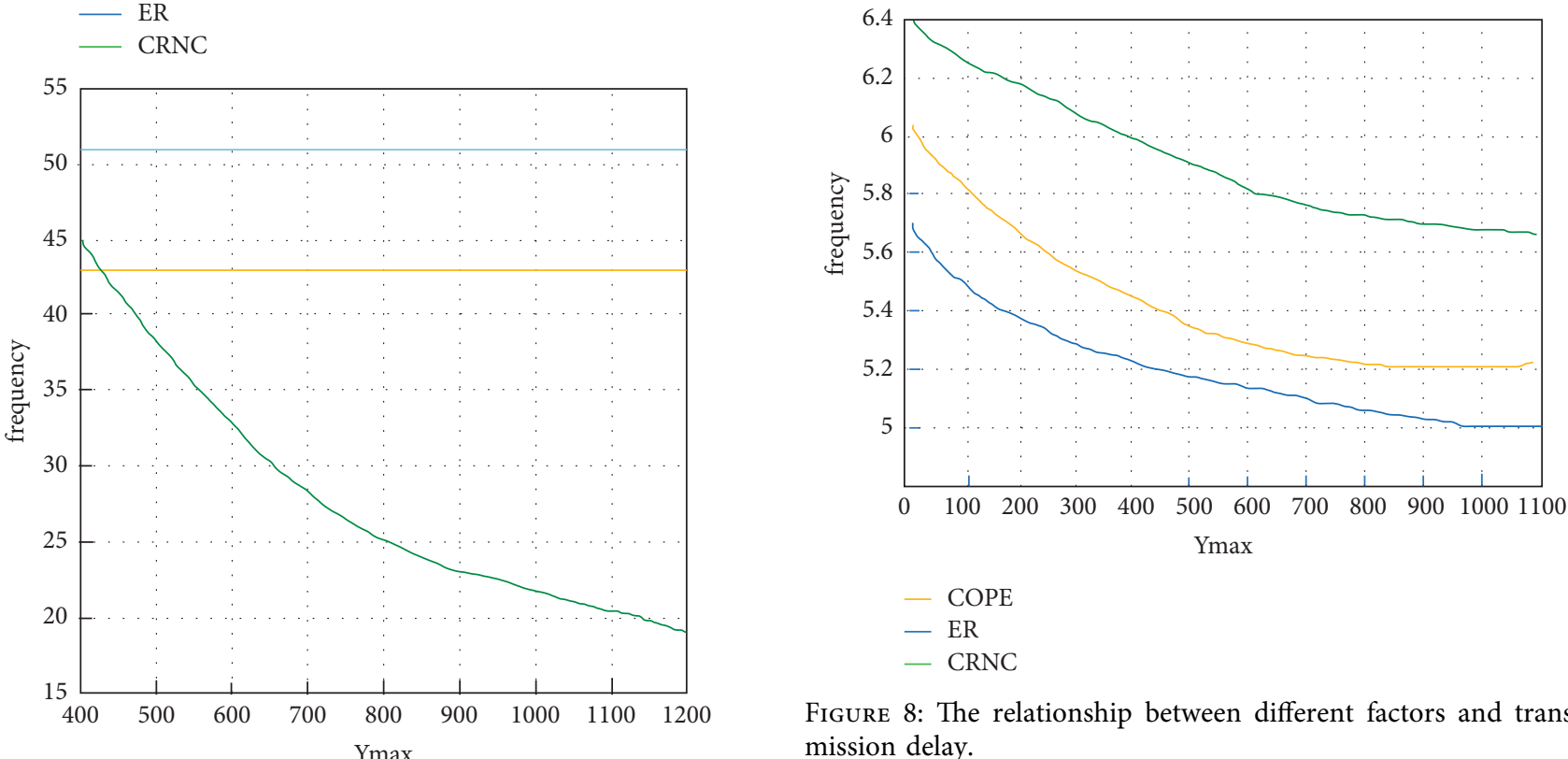

Figure 8: The relationship between different factors and transmission delay.

by the sink to receive complete source information, that is, the total energy overhead. Therefore, in a wireless ad hoc network with limited energy, network coding can achieve a balance between increasing network throughput gain and reducing energy consumption.

greatly reduces the average number of data packet exchanges of the intermediate node. The cost of transmission delay is relatively low. Obviously, the performance of the CRCN mechanism in terms of transmission delay is always better than that of the ER mechanism.

The simulation experiments can show that if it is assumed that network resources such as transmission bandwidth, node cache, and node computing power are sufficient, compared with the ER mechanism and COPE mechanism based on flooding replication, the CRNC mechanism based on network coding will slightly increase the transmission delay. The purpose of simulation is to simulate the limited transmission bandwidth in practical applications. The CRNC mechanism can pay a lower transmission delay cost in exchange for greatly reducing the total number of transmissions required

4.2. Reasons for Choosing to Optimize the Sum of Distances within the Class First. Separate the sum of intraclass distances from the sum of interclass distances for optimization, which is beneficial to treat the sum of intraclass distances and the sum of interclass distances equally. Because in the traditional feature extraction algorithm, optimizing the two at the same time, it is easy to cause the problem of unbalanced optimization. This is because in the data set, the sum of intraclass distances is generally much smaller than the sum of interclass distances. In this chapter, a series of verifications have been made to confirm the order of the sum of distances within the optimized class and the sum of distances between classes. Figure 9 shows the results on multiple data sets, and the accuracy varies by $30 \%$. 

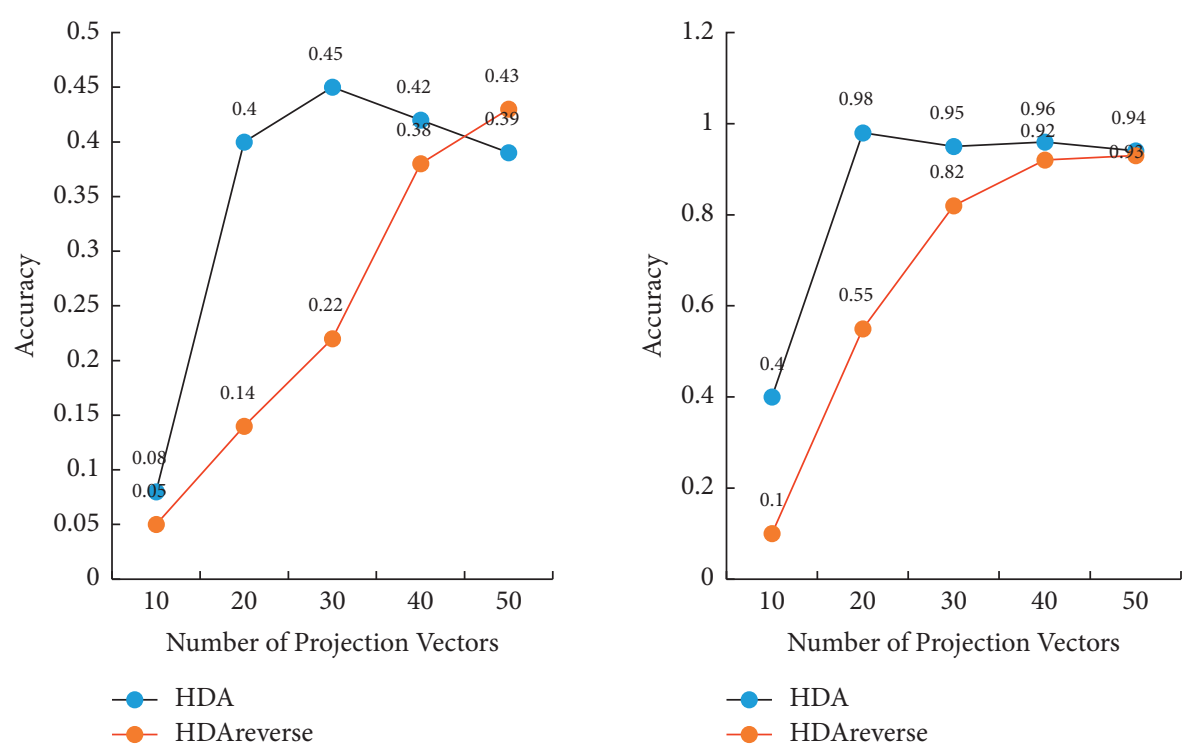

Figure 9: The accuracy of the two algorithms under different data sets.
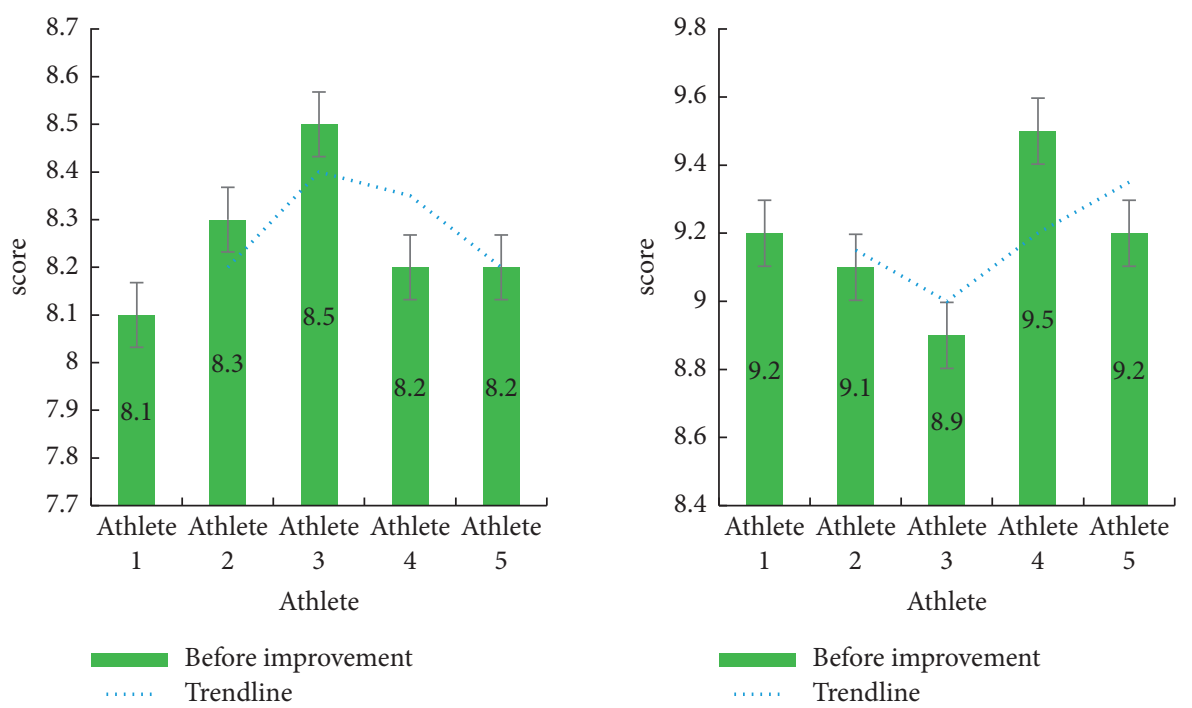

Figure 10: Comparison of performance scores of aerobics before and after improvement of athletes.

By comparing two different priority optimization strategies, comparing the recognition accuracy of the algorithm and the feature dimension selected at the best recognition rate, we found that the performance of minimizing the sum of intraclass distances first is far better than that of maximizing the sum of interclass distances first. We believe that the reason for this result is that the sum of distances between classes is much greater than the sum of distances within classes. After maximizing the sum of interclass distances, the new training sample set obtained according to the best projection matrix has been biased towards the optimal result of the sum of interclass distances. However, the sum of the distances within the class is not large enough, so it cannot play a good optimization role in optimization, which leads to a far drop in the recognition rate. Therefore, we chose to first minimize the sum of the distances within the class as the first step of our hierarchical discriminant analysis algorithm.

4.3. Before and after Performance Improvement. In order to explore the improvement of the performance of aerobics athletes in the wireless communication network and edge computing, this paper is designed to calculate the performance scores of five athletes before and after the improvement. The specific statistics are shown in Figure 10.

From the abovementioned scores of aerobics performance before and after improvement, it can be seen that the scores of athletes before improvement are 8.1, 8.3, 8.5, 8.2, and 8.2 , and the overall score is low. The improved athlete 
scores are 9.2, 9.1, 8.9, 9.5, and 9.2, and the overall score has been greatly improved. The performance score of athletes has increased by an average of $13 \%$, which can greatly improve the performance of aerobics athletes.

\section{Conclusions}

This article mainly studies how to improve the performance of aerobics athletes. Through the introduction of wireless communication network technology and edge computing, data acquisition of aerobics athletes is carried out. Accurate collection and use of the computing power of edge computing to analyze and summarize the collected data to improve the performance of aerobics athletes is carried out. Finally, in the experimental part, an experimental analysis is carried out on the accuracy of data collection and the related parameters of data collection, and the relevant data is summarized in the analysis part.

\section{Data Availability}

No data were used to support this study.

\section{Conflicts of Interest}

The author declares no conflicts of interest.

\section{References}

[1] Y. Ma, "Research on the arrangement and visual design of aerobics under the new situation," International Core Journal of Engineering, vol. 5, no. 9, pp. 170-173, 2019.

[2] R. M. Cunha, J. Vilaça-Alves, M. V. Noleto et al., "Acute blood pressure response in hypertensive elderly women immediately after water aerobics exercise: a crossover study," Clinical and experimental hypertension (New York, N.Y.: 1993), vol. 39, no. 1, pp. 17-22, 2017.

[3] T. Taleb, K. Samdanis, B. Mada, H. Flinck, S. Dutta, and D. Sabella, "On multi-access edge computing: a survey of the emerging 5G network edge cloud architecture and orchestration," IEEE Communications Surveys \& Tutorials, vol. 19, no. 3, pp. 1657-1681, 2017.

[4] Z. Ke, Y. Mao, S. Leng, Y. He, and Y. Zhang, "Mobile-edge computing for vehicular networks: a promising network paradigm with predictive off-loading," IEEE Vehicular Technology Magazine, vol. 12, no. 2, pp. 36-44, 2017.

[5] L. He, K. Ota, and M. Dong, "Learning IoT in edge: deep learning for the Internet of Things with edge computing," IEEE Network, vol. 32, no. 1, pp. 96-101, 2018.

[6] W. Yu, F. Liang, X. He et al., "A survey on the edge computing for the Internet of Things," IEEE Access, vol. 6, no. 99, pp. 6900-6919, 2018.

[7] S. Nastic, T. Rausch, O. Scekic et al., "A serverless real-time data analytics platform for edge computing," IEEE Internet Computing, vol. 21, no. 4, pp. 64-71, 2017.

[8] X. Chen, Q. Shi, L. Yang, and J. Xu, "ThriftyEdge: resourceefficient edge computing for intelligent IoT applications," IEEE Network, vol. 32, no. 1, pp. 61-65, 2018.

[9] M. Ebrahimi, T. N. Guilan-Nejad, and A. F. Pordanjani, "Effect of yoga and aerobics exercise on sleep quality in women with Type 2 diabetes: a randomized controlled trial," Sleep Science, vol. 10, no. 2, pp. 68-72, 2017.
[10] S. Tonstad, P. Herring, J. Lee, and J. D. Johnson, “Two physical activity measures: paffenbarger physical activity questionnaire versus aerobics center longitudinal study as predictors of adultonset type 2 diabetes in a follow-up study," American Journal of Health Promotion, vol. 32, no. 4, pp. 1070-1077, 2018.

[11] N. Le Chau, T.-P. Dao, and V. A. Dang, "An efficient hybrid approach of improved adaptive neural fuzzy inference system and teaching learning-based optimization for design optimization of a jet pump-based thermoacoustic-Stirling heat engine," Neural Computing \& Applications, vol. 32, no. 11, pp. 7259-7273, 2020.

[12] Y. Yan, X. Sun, B. Li, and X. Wang, "Construct a teaching system combining image linguistics and multimedia technology," Wireless Communications and Mobile Computing, vol. 2021, Article ID 6699010, 11 pages, 2021.

[13] E. Ahmed, A. Ahmed, I. Yaqoob et al., "Bringing computation closer toward the user network: is edge computing the solution?" IEEE Communications Magazine, vol. 55, no. 11, pp. 138-144, 2017.

[14] K. Kaur, S. Garg, G. S. Aujla, N. Kumar, J. J. P. C. Rodrigues, and M. Guizani, "Edge computing in the industrial Internet of Things environment: software-defined-networks-based edgecloud interplay," IEEE Communications Magazine, vol. 56, no. 2, pp. 44-51, 2018.

[15] L. Yang, C. Xu, Y. Zhan, Z. Liu, J. Guan, and H. Zhang, "Incentive mechanism for computation offloading using edge computing: a Stackelberg game approach," Computer Networks, vol. 129, pp. 399-409, 2017.

[16] R. Wang, J. Yan, D. Wu, H. Wang, and Q. Yang, "Knowledgecentric edge computing based on virtualized D2D communication systems," IEEE Communications Magazine, vol. 56, no. 5, pp. 32-38, 2018.

[17] B. P. Rimal, D. Pham Van, and M. Maier, "Mobile-edge computing versus centralized cloud computing over a converged FiWi access network," IEEE Transactions on Network and Service Management, vol. 14, no. 3, pp. 498-513, 2017.

[18] S. Wang, Y. Zhao, L. Huang, J. Xu, and C.-H. Hsu, "QoS prediction for service recommendations in mobile edge computing," Journal of Parallel and Distributed Computing, vol. 127, pp. 134-144, 2017.

[19] X. Lyu, H. Tian, L. Jiang et al., "Selective offloading in mobile edge computing for the green Internet of Things," IEEE Network, vol. 32, no. 1, pp. 54-60, 2018.

[20] T. A. Simakova, A. A. Zharkikh, and N. Ankudinov, "Experimental study of the influence of self-realization of students in sports activities on the tendency to cyber dependence in University Education Context," Psychology and Law, vol. 10, no. 2, pp. 140-150, 2020.

[21] Y. Cao, H. Song, O. Kaiwartya et al., "Mobile edge computing for big-data-enabled electric vehicle charging," IEEE Communications Magazine, vol. 56, no. 3, pp. 150-156, 2018.

[22] M. Gusev and S. Dustdar, "Going back to the roots-the evolution of edge computing, an IoT perspective," IEEE Internet Computing, vol. 22, no. 2, pp. 5-15, 2018.

[23] F. Qiang and N. Ansari, "Application aware workload allocation for edge computing based IoT," IEEE Internet of Things Journal, vol. 5, no. 3, pp. 2146-2153, 2018.

[24] L. Hong, Y. Zhang, and Y. Tao, "Blockchain-enabled security in electric vehicles cloud and edge computing," IEEE Network, vol. 32, no. 3, pp. 78-83, 2018.

[25] K. Zhang, S. Leng, Y. He, S. Maharjan, and Y. Zhang, "Cooperative content caching in $5 \mathrm{G}$ networks with mobile edge computing," IEEE Wireless Communications, vol. 25, no. 3, pp. 80-87, 2018. 